\title{
Fístula broncovascular - complicação de cateter venoso central percutâneo em neonato
}

\author{
Bronchovascular fistula - complication of \\ percutaneous central venous catheter in a neonate
}

Cláudio D‘Elia' ${ }^{1}$, Marta S. Correia ${ }^{2}$, Suzana D. de Oliveira ${ }^{3}$, Nilse M.M. Barbosa ${ }^{4}$

\section{Resumo}

Objetivos: relatar um caso em que ocorreu falso trajeto de cateter venoso central, com fístula vásculo-pulmonar e graves conseqüências respiratórias correlatas em recém-nascido. Revisar a literatura sobre as complicações respiratórias e não respiratórias relacionadas à introdução de cateteres venosos centrais percutâneos em crianças.

Descrição: dados clínicos evolutivos e diagnósticos foram obtidos após revisão do prontuário. O recém-nascido prematuro permaneceu em UTI neonatal após o parto para tratamento de doença da membrana hialina leve e de infecção manifestada posteriormente. No dia seguinte à introdução percutânea do cateter central, para a administração de nutrição parenteral, iniciou desconforto respiratório que progrediu rapidamente. Necessitou de ventilação mecânica para estabelecer troca gasosa adequada. A verificação da trajetória do cateter com o auxílio de contraste radiológico revelou a presença da complicação.

Comentários: não são raras as complicações decorrentes da inserção de cateteres centrais, sendo a infecção a mais comum. É importante que os profissionais responsáveis pelos cuidados desses pacientes conheçam as várias outras complicações menos freqüentes, como a trombose vascular e a migração do cateter, com lesões de órgãos e coleções extravasculares de líquidos. Em recém-nascidos, existe apenas um único relato de complicação semelhante à verificada em nosso paciente. Sua raridade pode ter determinado dificuldades para o diagnóstico imediato. São enfatizados os aspectos relacionados aos cuidados, após a introdução destes cateteres, que poderiam facilitar o reconhecimento precoce destas complicações.

J Pediatr (Rio J) 2002; 78 (4): 347-50: fístula brônquica, cateterismo, complicações, recém-nascidos.

1. Mestre em Medicina pela UFRJ. Especialista em Pneumologia Pediátrica pela SBPT/SBP. Médico do IPPMG/UFRJ.

2. Enfermeira da TIPE - Hospital Panamericano.

3. Médica Intensivista da TIPE - Hospital Panamericano.

4. Chefe da UTI neonatal TIPE - Hospital Panamericano.

Artigo submetido em 25.02.02, aceito em 22.05.02.

\begin{abstract}
Objectives: to present a case of central venous line misplacement with bronchial fistula and severe respiratory consequences in a newborn. To review the literature concerning respiratory and nonrespiratory complications related to the introduction of percutaneously placed central venous lines in children.
\end{abstract}

Report: evaluative and diagnostic data were described after patient chart review. A premature newborn was admitted to a NICU after delivery for treatment of low-grade hyaline membrane disease and infection manifested afterwards. One day after the introduction of the percutaneously placed central venous catheter, in order to provide parenteral nutrition, respiratory distress began with rapid progression. Mechanical ventilation was required to establish proper gas exchange. The complication was revealed after checking the catheter course using radiographic contrast.

Comments: complications associated with central venous catheter insertion are not rare and infection is the most frequent one. It is essential that caretakers be aware of the several less frequent complications, such as vascular thrombosis and catheter misplacement with organ injury and extravascular fluids collections. Only one description related to this kind of complication verified in our patient was found in newborns. This unique characteristic may have hindered a prompt diagnosis. Care related aspects, after catheter insertion, that could make easier the prompt recognition of these complications are emphasized.

J Pediatr (Rio J) 2002; 78 (4): 347-50: bronchial fistula, catheterization, adverse effects, newborn. 
vez mais em UTIs neonatais. Suas vantagens estão relacionadas à possibilidade de suporte nutricional via parenteral, à administração mais segura de líquidos, à possibilidade de monitorização hemodinâmica e à maior facilidade em mantê-los, evitando o estresse e o desconforto de repetidas punções. Os cateteres venosos centrais de punção percutânea (PICC) têm sido utilizados no tratamento de recémnascidos (RN) há quase três décadas, particularmente nos prematuros que necessitam de acesso venoso prolongado para receber nutrição parenteral. A descrição inicial do uso de PICC em neonatos foi feita por Shaw, em 1973 ${ }^{1}$. Desde então, o uso desses cateteres nessas crianças tem sido avaliado prospectivamente, sendo demonstrada a sua efetividade em assegurar um acesso venoso central prolonga$\mathrm{do}^{2}$.

Os autores descrevem um caso de fístula bronco-vascular, com graves repercussões para o RN, decorrente da migração extravascular do PICC (Per-Q-Cath; Bard Access Systems, Salt Lake City, Utah). Esta complicação é extremamente rara, e, após revisão sobre o assunto, encontramos em recém-nascidos apenas um relato prévio de fístula brônquio-arterial (a partir de ramificação da artéria pulmonar) $^{3}$, diferindo deste, cuja fístula é brônquio-venosa (a partir da veia cava superior).

\section{Descrição do caso}

RN com nascimento prematuro após cesariana, devido à pré-eclâmpsia, com apgar $8 / 8$, capurro somático $(\mathrm{CS})=35$ semanas, e peso de nascimento de $2.410 \mathrm{~g}$, foi admitido na UTI neonatal com quatro horas de vida para tratamento de desconforto respiratório. A radiografia torácica era compatível com doença da membrana hialina (DMH) leve. Necessitou de oxigenoterapia (oxyhood) $\mathrm{com} \mathrm{FiO}_{2}$ baixas até o terceiro dia de vida. No dia seguinte, apresentava hipoatividade e leucograma anormal $(\mathrm{NT}=5.964, \mathrm{NJ}=924$, rel=0,15). $\mathrm{O}$ abdômen encontrava-se distendido, portanto foi decidido iniciar nutrição parenteral (NP) parcial através de acesso venoso periférico. A dieta enteral (LHO), com baixos volumes, foi iniciada no quinto dia de vida, e a NP foi suspensa no sétimo.

No oitavo dia, apresentou distúrbio de perfusão, febre, e a radiografia torácica revelou infiltrado parahilar direito. Foram iniciados antimicrobianos parenterais, aminas vasoativas e oxigenoterapia (OxyHood 0,3). A dieta foi suspensa. Não apresentava anormalidades gasométricas ou do padrão respiratório, e a administração de $\mathrm{O}_{2}$ foi interrompida após 24h. A antibioticoterapia foi modificada após $72 \mathrm{~h}$ devido ao resultado da cultura de sangue (enterobacter). No décimo segundo dia de vida, foi reiniciada NP através de PICC, introduzido no mesmo dia em veia do segmento cefálico. No dia seguinte, foram observados desconforto respiratório e quedas freqüentes da Sat. $\mathrm{O}_{2}$. Evoluiu com insuficiência respiratória em menos de 24 horas, sendo intubado para o início da ventilação mecânica. As radiografias subseqüentes apresentavam infiltrados bilaterais, inici-

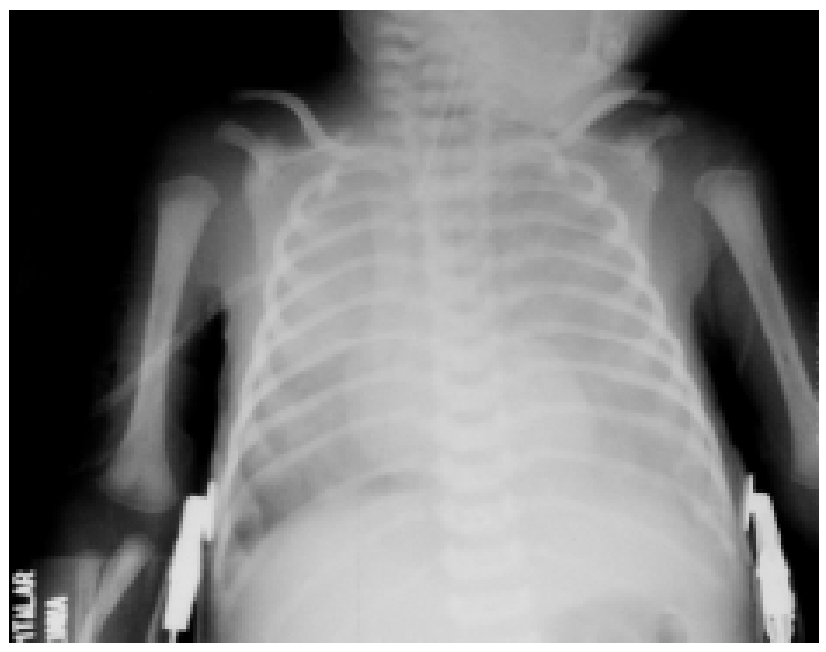

Figura 1 - Vinte e quatro horas após a introdução do PICC. Insuficiência respiratória. Infiltrado bilateral difuso

almente mais pronunciados à esquerda, e depois hipotransparência difusa. $\mathrm{O}$ ecocardiograma era normal. Devido à piora do quadro pulmonar, a broncoscopia foi realizada no décimo quinto dia de vida para obtenção do lavado broncoalveolar. Havia grande quantidade de líquido de baixa viscosidade, semelhante à NP, em vias aéreas e, portanto, foi injetado contraste (Optiray 320) no cateter venoso (PICC), sendo evidenciada a fístula bronco-vascular. Os diagnósticos prováveis de pneumonia lipídica e edema pulmonar iatrogênico foram estabelecidos.

Após a retirada do cateter, houve rápida melhora clínica e radiológica, com extubação após dois dias.

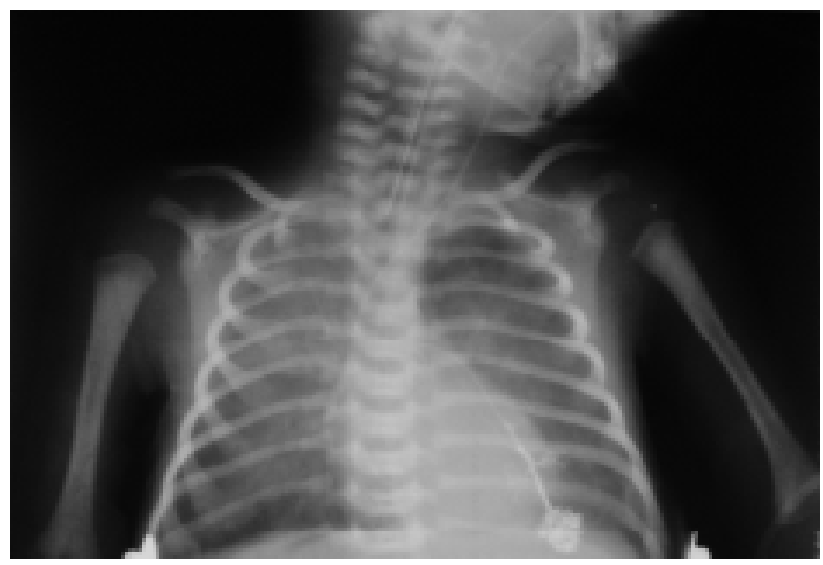

Figura 2 - Contraste radiográfico revelando a fístula broncovascular (broncograma da divisão do lobo inferior esquerdo) 


\section{Discussão}

O PICC é definido como um cateter que é introduzido percutaneamente através de uma veia periférica até sua ponta alojar-se em uma veia central. Cateteres intravenosos periféricos (jelcos) são de inserção relativamente fácil, entretanto necessitam de trocas freqüentes devido à flebite ou infiltração, com dificuldades progressivas na obtenção de novos sítios e na manutenção dos acessos. A administração de líquidos e medicações endovenosas por estes cateteres podem causar, ocasionalmente, lesão tissular, necrose e, por vezes, descamação epidérmica intensa ${ }^{4}$.

O sucesso na inserção do PICC em recém-nascidos é menor do que em crianças mais velhas. No entanto, radiologistas utilizando técnicas de imagem como o ultra-som ou a fluoroscopia para guiar a colocação do cateter podem obter altas taxas de sucesso, variando entre 67 e $94 \%$ na literatura ${ }^{5,6}$. Problemas mecânicos, como as lacerações, podem ocorrer em 16 a $27 \%^{5}$. Deslocamento, devido à dificuldade de fixação no paciente, e oclusão do cateter também podem ocorrer. Alguns estudos, que avaliaram a colocação de PICC em crianças, verificaram taxas de complicações relativamente baixas ${ }^{6-8}$ e comparativamente menores em relação aos cateteres inseridos cirurgicamente ${ }^{9}$. Contudo, algumas complicações graves associadas à introdução deste tipo de cateter foram relatadas: derrames pleurais e pericárdicos, tamponamento cardíaco, perfuração do miorcárdio, arritmias cardíacas, migração do cateter para o cérebro $^{10}$, trombose vascular ${ }^{11}$, bacteremia, endocardite, sepse $^{11}$, embolização pulmonar após fratura e migração do cateter ${ }^{12}$ e pneumonite causada pela NP através de ramos da artéria pulmonar ${ }^{3}$.

A principal indicação para a introdução do PICC é a necessidade de acesso venoso por período maior do que três dias. A decisão de introduzir o PICC neste paciente foi tomada devido à necessidade de acesso venoso estável e prolongado para a administração de antimicrobianos e nutrição parenteral.

A localização central é definida se a ponta do cateter reside na veia cava superior, átrio direito ou veia cava inferior, acima ou ao nível do diafragma ${ }^{13}$. Às vezes não é possível a inserção do cateter até uma localização central, permanecendo a ponta ocasionalmente nas veias braquiocefálica, jugular, axilar ou safena. Isso pode ocorrer em até $33 \%$ das punções ${ }^{5}$. Algumas razões para esta dificuldade incluem vasoespasmo, tortuosidade da veia ou a presença de válvulas venosas. Essas localizações (midline) estão associadas a um maior número de complicações, provavelmente por uma combinação de fatores como o tamanho do vaso, fluxo de sangue venoso, turbulência do fluxo e lesão endotelial $^{13}$. A National Association of Vascular Access Networks recomenda que o posicionamento ideal dos PICCs seja no terço inferior da veia cava superior, próximo à junção com o átrio direito, se inseridos perifericamente a partir da cabeça ou da extremidade superior. A posição da ponta do cateter deve ser verificada através de exame radiológico (spot), contudo, não sabemos se houve esta preocupação neste caso, pois nada foi relatado no prontuário. Os exames radiológicos realizados após o início das anormalidades respiratórias não revelam facilmente o percurso do cateter, entretanto isso poderia ser resolvido com a injeção de 1 a $2 \mathrm{ml}$ de contraste radiográfico ${ }^{14}$.

Migração da ponta do cateter parece ser um problema relativamente comum, e a monitorização, através de dois exames radiológicos semanais, é recomendada por outros autores ${ }^{10}$. Houve um curto período de tempo entre a introdução do PICC e o início dos sintomas respiratórios; além disso, a radiografia com contraste realizada logo após a broncoscopia revelou um longo caminho entre a perfuração da veia cava superior e o brônquio do lobo inferior esquerdo. Portanto, acreditamos que a migração extravascular tenha ocorrido durante o procedimento de inserção do PICC, e não por problemas relacionados à fixação do cateter no paciente. Se isso realmente aconteceu, houve falha na realização da técnica recomendada para a introdução dos PICCs nas veias do escalpo. A extensão da parte do cateter a ser inserida deve ser medida do local de punção escolhido até dois dedos abaixo da fúrcula esternal ${ }^{14}$.

Perfurações vasculares e brônquicas já foram relatadas previamente, também com cateteres siliconizados ${ }^{3}$. No único caso em RN, as perfurações provavelmente ocorreram em um ramo da artéria pulmonar após migração do cateter e no brônquio fonte direito. $\mathrm{O}$ desfecho dessa grave complicação foi bom, logo após a remoção do cateter ${ }^{3}, \mathrm{o}$ mesmo sendo verificado por nós.

A rápida resolução clínica e radiológica não reforça a hipótese de pneumonia lipídica após a inadvertida administração endobrônquica da emulsão de lipídeos. A realização de tomografia computadorizada poderia ser útil para demonstrar as típicas consolidações de baixa densidade, com unidades Hounsfield negativas, indicando a presença de depósitos lipídicos ${ }^{15}$.

O uso de PICCs é cada vez mais freqüente nas unidades de terapia intensiva, quando veias periféricas não são visualizadas ou palpadas, e quando não é obtido sucesso após punções periféricas, em situações em que há necessidade de acesso venoso prolongado. A verificação da posição da ponta do cateter, $\operatorname{logo}$ após a punção e durante a sua permanência na veia, poderia evitar esse tipo de complicação, ou permitir a identificação precoce da migração ou extravasamento, para a adoção de medidas apropriadas.

\section{Referências bibliográficas}

1. Shaw JL. Parenteral nutrition in the management of sick low birth weight infants. Pediatr Clin North Am 1973;20:333-58.

2. Frey AM. Pediatric peripherally inserted central catheter program report: a summary of 4,536 catheter days. J Intraven Nurs 1995; 18:280-91.

3. Martinez-Tallo E, Hernandez-Rastrollo R, Rodino EA, RuizCabal JE. Parenteral nutrition infused by epicutaneous catheter: pulmonary complication. J Perinat Med 1995;23:223-7. 
4. Lesser E, Chhabra R, Brion LP, Suresh BR. Use of midline catheters in low birth weight infants. J Perinatol 1996;16:205-7.

5. Hogan MJ. Neonatal vascular catheters and their complications. Radiol Clin North Am 1999;37:1109-25.

6. Crowley JJ, Pereira JK, Harris LS, Becker CJ. Peripherally inserted central catheters: experience in 523 children. Radiology 1997;204:617-21.

7. Frey AM. PICC complications in neonates and children. Journal Vascular Access Devices Spring 1999;4:17-26.

8. Donaldson JS, Morello FP, Junewick JJ, O’Donovan JC, LimDunham J. Peripherally inserted central venous catheters: USguided vascular access in pediatric patients. Radiology 1995;197:542-4.

9. Shulman RJ, Pokorny WJ, Martin CG, Petitt R, Baldaia L, Roney D. Comparison of percutaneous and surgical placement of central venous catheters in neonates. J Pediatr Surg 1986;121:348-50.

10. Nadroo AM, Lin J, Green RS, Magid MS, Holzman IR. Death as a complication of peripherally inserted central catheters in neonates. J Pediatr 2001;138:599-601.

11. Hruszkewycz V, Holtrop PC, Batton DG, Morden RS, Gibson P, Band JD. Complications associated with central venous catheters inserted in critically ill neonates. Infect Control Hosp Epidemiol 1991;12:544-8.
12. Kossoff EH, Poirier MP. Peripherally inserted central venous catheter fracture and embolization to the lung. Pediatr Emerg Care 1998;14:403-5.

13. Racadio JM, Doellman DA, Johnson ND, Bean JA, Jacobs BR. Pediatric peripherally inserted central catheters: complication rates related to catheter tip location. Pediatrics 2001;107:28.

14. Racadio JM, Johnson ND, Doellman DA. Peripherally inserted central venous catheters: success of scalp-vein access in infants and newborns. Radiology 1999;210:858-60.

15. Joshi RR, Cholankeril JV. Computed tomography in lipid pneumonia. J Comput Assist Tomogr 1985;9:211-3.

Endereço para correspondência:

Dr. Cláudio D'Elia

Rua Prof. Coutinho Frois, 334 - ap. 202 - Barra da Tijuca

CEP 22620-360 - Rio de Janeiro, RJ

E-mail: claudiodelia@uol.com.br 